\title{
Brightness interactions between rods and cones
}

\author{
BRUCE DRUM \\ Department of Ophthalmology, George Washington University, Washington, D.C. 20037
}

\begin{abstract}
Two parafoveal test targets with different spectral compositions were matched in brightness to a fixed-luminance foveal reference target under scotopic adaptation conditions. The idea of the experiment was to find a reference luminance for which one of the matching test targets stimulated only rods while the other stimulated both rods and cones. If brightness was proportional to the linear sum of rod and cone responses, then the luminance of the matching rod+cone target would be predictably closer to rod threshold than would that of the rod target. The results were complicated by evidence that rod responses to the test targets selectively enhanced weak chromatic signals. Nevertheless, it was possible to show that cone activity never reduced the matching luminance as much as predicted by the additivity hypothesis, and sometimes even increased it. These findings suggest that cone activity can suppress brightness signals from rods.
\end{abstract}

The visual sensation of brightness must ultimately arise from responses of the rod and cone photoreceptors. It is therefore interesting to consider how rod and cone brightness contributions combine when both receptor types are active. Previous psychophysical studies of this question have yielded mixed results. Tests of lateral brightness interactions between rods and cones have shown that, under appropriate conditions, either receptor type can reduce the brightness contribution of the other (Hollins, 1971; Richards, Luria, \& Matteson, 1967; Walters, 1970; Willmer, 1949). These experiments, however, used nonoverlapping rod and cone stimuli, and hence their findings do not apply to single test targets that stimulate both receptor types. Three studies of rod-cone brightness interactions have used spatially coincident rod and cone stimuli, but their results are far from clear. Clarke (1960) found gross failures of heterochromatic luminance additivity, but could not rule out the possibility that they were due to cone-cone interactions. Palmer (1976), on the other hand, concluded, from an analysis of brightness matching data, that rod-mediated and cone-mediated brightness components were linearly additive, but his model does not fit the data well enough to preclude significant additivity failures. Most recently, Spillmann and Breitmeyer (1977) failed to find large rod-cone brightness interactions, but they did show that bright-

Supported by HIH Grant EY01672 and by a grant from Research to Prevent Blindness, Inc. I am grateful to John McCann and Michael Breton for their comments on an earlier version of the manuscript, and to Fareed Armaly and William Huppert for technical assistance. This paper contains material presented at the 1977 annual meeting of the Optical Society of America, Toronto, Canada. Requests for reprints should be sent to Bruce Drum, Department of Ophthalmology, George Washington University, 2150 Pennsylvania Avenue, N.W., Washington, D.C. 20037. ness discrimination in the dark-adapted parafovea is impaired near the cone threshold.

The present paper further investigated rod and cone contributions to the brightness of homogeneous stimuli. The main experiment was designed to compare the brightness of a parafoveal target that stimulated both rods and cones with the linear sum of its rod and cone brightness components. This experiment was complicated by the unexpected observation that cone activity was evident at lower luminances when the test target was visible to both rods and cones than when it was visible to cones alone. Nevertheless, it was possible to show that when rods and cones both responded to the test target, the cone response reduced the rod brightness component.

\section{EXPERIMENT 1 BRIGHTNESS MATCHES}

This experiment tested the effects of cone activity on brightness sensations from rods by matching the brightness of two parafoveal test targets to that of a fixed-luminance foveal reference target under scotopic adaptation conditions. One test target was unfiltered tungsten light, and the other was the same light filtered with a band-pass glass filter. When viewed photopically, the unfiltered target appeared yellowish white and the filtered target appeared bluish green. Although these colors did not persist under the conditions of the experiment, for convenience I will refer to the unfiltered target as the "white" target and the filtered target as the "green" target.

The spectral distributions of the test targets were such that rod threshold was below cone threshold for both of them, but rod and cone thresholds were about $.5 \mathrm{log}$ unit farther apart for the green target than for the white target. There was thus a small brightness range for which the luminance of the green target was 
below cone threshold, but that of the white target was above cone threshold; that is, the green target stimulated only rods, while the white target stimulated both rods and cones. The experimental task was to equate the brightness of the white and green targets (by matching both targets to the same reference) within this critical brightness range, and to determine how far the resulting luminances were above rod threshold.

Figure 1 schematically illustrates the relative positions of the threshold and brightness matching luminances for the white and green targets, normalized with respect to cone threshold. The double-headed vertical arrows indicate the luminance differences between the brightness matches and rod thresholds for the white and green test targets. If brightness were determined by rod activity alone, these two differences should clearly be equal. However, the cone activity in the white target condition produces an additional brightness signal that can be expected to influence the overall brightness match. For example, if the rod-and cone-mediated brightness components added together linearly, the matching luminance would be correspondingly closer to the rod threshold than that of a comparable match mediated by rods alone. Alternatively, a luminance difference between the brightness match and rod threshold that was greater for the rod + cone condition than for the rod condition would imply that cone activity had suppressed the rod-mediated brightness component.

\section{Methods}

Apparatus and Subjects. The experiments were performed with a Tübingen perimeter. All targets were superposed on a uniform, steady, $-2.5 \log \mathrm{cd} / \mathrm{m}^{2}$ background. The luminance of this background was high enough to adapt rods but not cones. The purpose of the background was to reduce the rod/cone response ratio, thus making the experiment more sensitive to the effects of near-threshold cone signals. The background, the reference target, and the white test target were unfiltered tungsten light, with color temperatures of $3,000^{\circ}, 3,000^{\circ}$, and $3,400^{\circ}$, respectively. The green test target had a dominant wavelength of $498 \mathrm{~nm}$ and an excitation purity of $80 \%$. A $9,900-\mathrm{cd} / \mathrm{m}^{2} \mathrm{cool}$ white fluorescent light that covered the entire central field provided preadaptation for dark-adaptation measurements. The reference and test targets all had durations of $1 \mathrm{sec}$ and diameters of $1.1 \mathrm{deg}$ of visual angle. The subjects fixated the center of a diamond-shaped array (2-deg diagonals) of four dim, red 10-min spots. Two adult males with normal vision served as subjects. The testing position was $2 \mathrm{deg}$

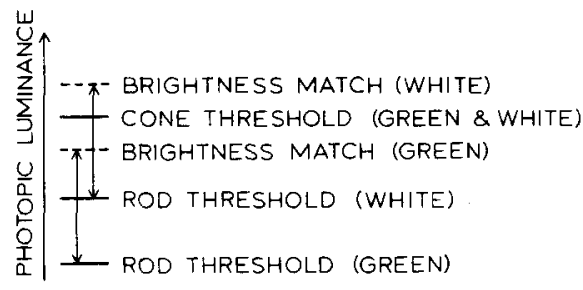

Figure 1. Luminance relationships of the various thresholds and brightness matches of Experiment 1. See full explanation in text. in the temporal field for one subject (F.A.) and $2 \mathrm{deg}$ in the nasal field for the second subject (B.D.). As mentioned before, the reference target was positioned foveally for both subjects. Pupil size was uncontrolled for both subjects, but pupil size changes could be safely assumed to be negligible for all relevant observations.'

Calibration. The photopic luminances of the unfiltered tungsten stimuli and the fluorescent preadapting light were all calibrated by making minimally distinct border matches (Wagner $\&$ Boynton, 1972) to the comparison field of a Macbeth illuminometer. The relative photopic luminances of the green and white test targets were arbitrarily equated at their cone plateau thresholds, and their relative scotopic luminances were equated at their rod thresholds. This procedure was considered more appropriate than assuming standard luminosity functions, because threshold was the relevant sensitivity measure and because the two subjects showed small but persistent spectral sensitivity differences. These differences were reflected in the effective optical densities of the green-target filter, computed from the cone plateau and rod thresholds. The average photopic and scotopic densities were, respectively, $1.43 \pm$ .03 and $1.05 \pm .03$ for Subject F.A. and $1.53 \pm .03$ and $1.00 \pm .06$ for Subject B.D.

Experimental procedures. Each experimental session included measurements of cone threshold for the reference target and both cone and rod thresholds for the two test targets. All of these thresholds were obtained by the following descending limits procedure. The experimenter presented the target at 3-5-sec intervals, starting at a target luminance just high enough to be easily detectable. After each positive response by the subject, the luminance decreased by $.1 \log$ unit, but after each negative response it remained the same. Threshold was considered to be the luminance that elicited three successive negative responses. The median of three to five such measurements was taken as the threshold for that session. ${ }^{2}$

As noted earlier, all thresholds were measured in the presence of the scotopic background. Under these conditions, however, cone thresholds for the test targets could not be measured directly because the rods were more sensitive. Cone threshold estimates for the two test targets were therefore obtained at the start of each session during the cone plateau of a dark-adaptation curve. After $4 \mathrm{~min}$ of preadaptation, thresholds were measured by the procedure described above at intervals of about $30 \mathrm{sec}$. Alternating the green- and white-target thresholds made it possible to gather data for both targets in a single dark-adaptation period. The two resulting functions typically contained obvious plateaus starting at 4-6 min and extending to 9-13 min after the onset of dark adaptation. Median thresholds for the session were taken from the data points which fell unambiguously on these plateaus.

After completing the cone plateau thresholds, the subject continued to dark adapt for an additional $30 \mathrm{~min}$, time enough for his rods to fully recover from the preadapting light. The remaining thresholds and the brightness matches were then measured.

An essential part of the experiment was to find a reference brightness for which the luminance of the matching white target was above the cone plateau threshold and that of the matching green target was below. To ensure that this requirement was met for at least one brightness level, matches were made up for up to four levels separated by $.2 \log$ unit steps of reference luminance.

Before each brightness match, preliminary tests established a range of several test target luminances, .1 log unit apart, that included the match. Equal numbers of test targets at each luminance were then presented in random order, always simultaneously with the reference. After each trial, the subject indicated whether the test target was brighter than (1), equal to (0), or dimmer than $(-1)$ the reference. The responses for each level were then totaled, and the match point was interpolated.

At the end of each of Subject B.D.'s sessions, an extra darkadaptation curve was measured. During the cone plateau of this curve, white-target brightness matches were measured at all reference levels for which the previous green-target matches had been below the cone plateau threshold. The purpose of this final step was to provide a direct comparison of "pure-cone" and "pure-rod" 
matches. ${ }^{3}$ Subject F.A. completed no cone plateau matches in his regular sessions, but he did make both a green-target (rod) match and a white-target cone plateau match at a single reference level in a later, abbreviated session.

\section{Results}

Figure 2 shows averaged results (three sessions) for Subject F.A. Part a shows the log luminances of all thresholds and brightness matches for both the reference and test targets, normalized at the 2-deg cone plateau threshold, as previously depicted in Figure 1. In part $b$, the differences between the log brightness matches and log rod thresholds are replotted in histogram form for the green (rod) and white (rod + cone) test targets. Note that the heights of these histogram bars are analogous to the lengths of the vertical arrows in Figure 1. The key result in Figure 2 is that, although the white target brightness match exceeded the cone plateau threshold by more than $.2 \log$ unit, it was just as far above rod threshold as the green-target match. Adding a cone stimulus to a constant rod stimulus thus produced no net brightness increase.

Ekman and Gustavsson (1968) have shown that, for dark-adapted conditions, brightness is proportional to luminance for luminances within 1 log unit of threshold. Assuming that this result applies to the present conditions, it is easy to find the rod + cone match predicted by linear additivity if the relative brightness of rod and cone threshold signals are known (see Appendix for details). These relative threshold brightnesses can be estimated by matching pure-rod and pure-cone test targets to the same refer- (a)

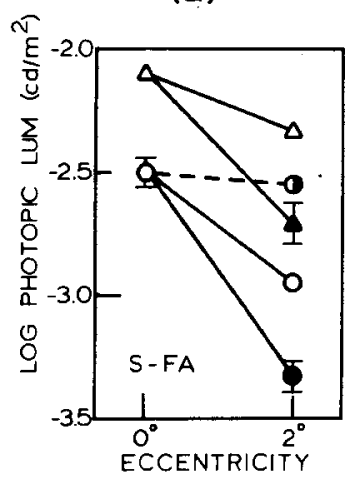

(b)

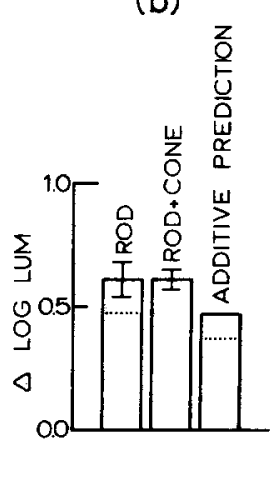

Figure 2. (a) Rod and cone thresholds (circles) and brightness matches (triangles) for green (filled symbols) and white (open symbols) targets. Data are averaged from three sessions for Subject F.A. Error bars indicate all standard errors exceeding .05 log unit. A dashed line connects the foveal reference threshold to the 2-deg cone plateau threshold. The relative luminances of the green and white test targets have been arbitrarily equated at the 2-deg cone plateau threshold (half-filled circle). (b) Difference between $\log$ brightness match and $\log$ rod threshold, taken from (a), for the green target (rods) and the white target (rods + cones). Error bars are standard errors of the differences. See text for discussion of additivity prediction and horizontal dotted lines. (a)

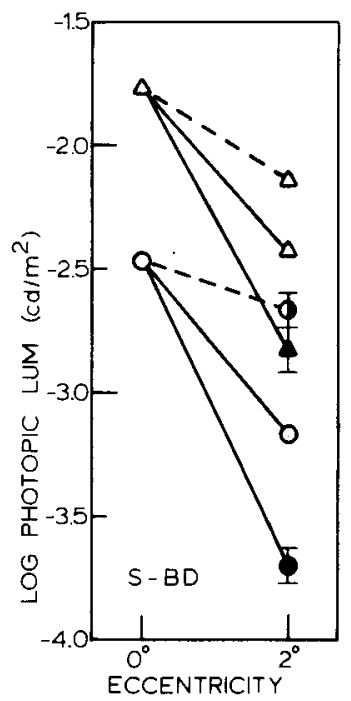

(b)

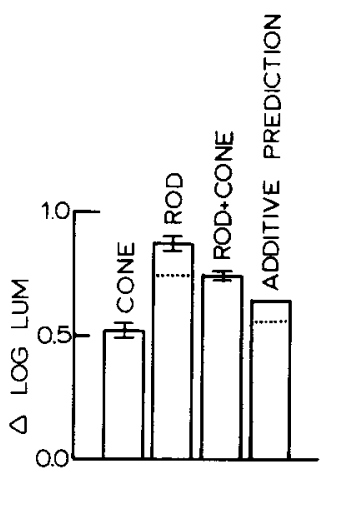

Figure 3. (a) Green- and white-target thresholds and brightness matches for Subject B.D. The uppermost 2-deg triangle is the average cone plateau brightness match, and is connected to the reference luminance by a dashed line. Other details are as in Figure 2a. (b) Difference between $\log$ brightness match and $\log$ threshold for the white cone plateau target (cones) and between $\log$ brightness match and $\log$ rod threshold for the green (rods) and white (rods + cones) targets. Other details are as in Figure $\mathbf{2 b}$.

ence. The ratios of the resulting pure-rod and purecone matching luminances to their respective threshold luminances should be inversely proportional to their threshold brightnesses. Subject F.A.'s pure-rod and pure-cone matches were nearly equidistant from threshold (within .1 log unit), suggesting that rod and cone threshold brightnesses were approximately equal. An additivity prediction assuming equal rod and cone threshold brightnesses was accordingly computed, and is plotted as the rightmost histogram bar in Figure 2 b. F.A.'s actual match clearly exceeds the prediction.

Figure 3 shows subject B.D.'s data, also averaged from three sessions. ${ }^{4}$ Cone plateau brightness matching data are included, since they are directly comparable to the rod brightness matching data (see experimental procedures section).

B.D.'s average white-target match was .13 log unit closer to rod threshold than his green-target match. Had he shown equal rod and cone threshold brightnesses, this match would have been consistent with additivity. Unlike F.A., however, B.D.'s cone plateau threshold appeared more than twice as bright as his rod threshold. (Assuming that the cone- and rodmediated brightnesses were both proportional to luminance, the log ratio of their threshold brightnesses is simply the height difference between the left two histogram bars in Figure 3b.) This extra cone brightness further lowered the predicted additive match to the level of the right-hand histogram bar in Figure 3b. 
B.D. also differed from F.A. in having a greater distance between his rod and cone plateau thresholds. A given cone brightness component should thus have had less relative effect on overall brightness for B.D. than for F.A., making B.D.'s data a less sensitive test of brightness additivity.

Two of F.A.'s brightness matches that were not included in Figure 2 suggested the probable mechanism for the additivity failures in Figures 2 and 3. The response vs. log luminance curves for these matches, plotted in Figure 4, showed nonmonotonic brightness changes with increasing luminance, and in both cases these reversals coincided with the cone plateau threshold. In other words, exceeding the cone plateau threshold appeared to reduce net brightness.

Figures 2 and 3 probably underestimate the extent of additivity failure between rod and cone brightness signals. Figure 5a shows the average distance from rod threshold of eight pairs of brightness matches (three for F.A. and five for B.D.) for which both the green- and white-target matches fell below the cone plateau threshold. The green-target matches in these cases averaged $.13 \mathrm{log}$ unit farther above threshold than the comparable white-target matches. A pairedobservation $t$ test $(\mathrm{df}=7)$ showed this difference to be significant at the .01 level. If the green-target matches in Figures 2 and 3 are assumed to be too high by the same margin, the green-target (rod) matches and the additivity predictions drop to the dotted lines in Figures $2 b$ and $3 b$.

\section{EXPERIMENT 2 CORROBORATION OF CONE THRESHOLDS}

Since the interpretation of the brightness matching experiment hinged on the position of cone threshold, it was desirable to know whether cone threshold was the same during the brightness matches as during the cone plateau measurements. In an attempt to answer this question, the long-term stability of the cone plateau was tested for three cases that were (a)

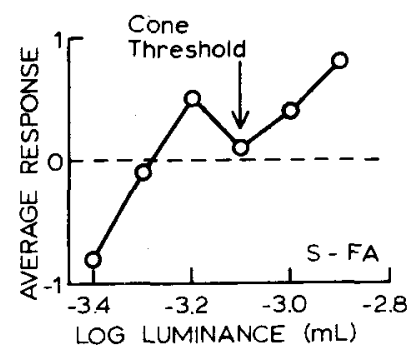

(b)

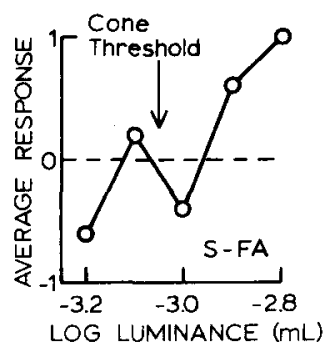

Figure 4. Nonmonotonic response functions for two brightness matches, Subject F.A. Horizontal dashed lines indicate the matches. Vertical arrows indicate the cone plateau thresholds. (a) White target. (b) Green target.
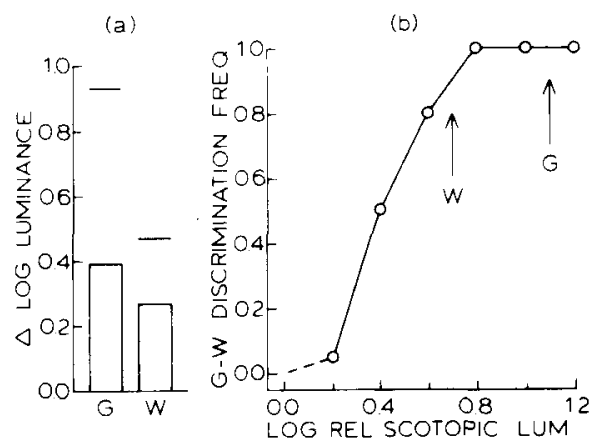

Figure 5. (a) Average differences (eight sessions) between log brightness matches and log rod thresholds for the green (G) and white (W) targets. All data are included for which the whitetarget brightness match fell below the cone plateau threshold. The horizontal lines above the histogram bars indicate the average relative luminances of the cone plateau thresholds. (b) Color discrimination frequency between green and white targets as a function of $\log$ relative scotopic luminance (re rod threshold), for Subject F.A. Vertical arrows indicate log cone plateau thresholds for the white $(W)$ and green $(G)$ targets.

similar to the brightness matching conditions, except that cones were more sensitive to the test target than were rods.

\section{Methods}

Dark-adaptation curves lasting at least $\mathbf{4 5}$ min were measured for green and white foveal targets and for a "red" target (Schott RG2 filter, dominant wavelength of $644 \mathrm{~nm}$ ) at the parafoveal test position. All other procedures and stimulus parameters were the same as for the cone plateau and rod thresholds in the brightness matching experiment. For all three cases, cone threshold remained well below rod threshold, even after the rods had fully adapted to the scotopic background.

\section{Results}

In every case, cone thresholds near the beginning of the cone plateau proved to be identical, within experimental error, to those measured after $45 \mathrm{~min}$ of dark adaptation. This result strongly suggested that cone pathways were equally adapted for the cone plateau thresholds and brightness matches of the brightness matching experiment.

\section{EXPERIMENT 3 COLOR DISCRIMINATION}

In conjunction with the surprising result shown in Figure 5a, both subjects noticed, during the brightness matching experiment, that the colors of the green and white targets were distinguishable well below the cone plateau threshold. Specifically, the green target remained recognizably greenish below the cone plateau, whereas the (slightly yellowish) white target became more achromatic. This observation was quantified with a forced-choice color-discrimination experiment. 


\section{Methods}

Twenty green and 20 white targets were presented at each of six luminance levels (re rod threshold), in .2 log unit steps from rod threshold to above cone plateau threshold. Adaptation and target parameters were the same as for the matching experiment. The presentation order was randomized so that the subject could predict neither the color nor the brightness of the next flash. After each flash, the subject responded " $X$ " or "O," respectively, if he thought the white or the green target had been presented. The proportions of correct responses to white and green targets were then pooled and adjusted for chance to obtain a discrimination frequency vs. $\log$ luminance function.

\section{Results}

Figure $5 \mathrm{~b}$ shows results of the color discrimination experiment for F.A. Discrimination departed from zero immediately above rod threshold, and was already well above .5 at the cone plateau threshold for the white target. ${ }^{5}$ An additional finding not apparent in the graph was that, except for the lowest level, all errors were made on the white target; that is, greentarget responses were always correct.

B.D.'s discrimination results (not shown) were nearly identical to those in Figure $5 b$ when normalized with respect to the green-target cone plateau threshold. B.D. showed slightly poorer discrimination than F.A. at his white-target cone plateau threshold (although still above .5), possibly because his green- and whitetarget cone plateau thresholds were $.15 \mathrm{log}$ unit farther apart when plotted relative to rod threshold.

\section{GENERAL DISCUSSION}

Both the brightness matching and color discrimination results in Figure 5 implied that cone activity persisted below the cone plateau threshold. The fact that such activity was not evident in control tests with foveal and red parafoveal targets indicated that it did not result from cone adaptive changes. Neither could it be dismissed as a measurement artifact, in view of the low criterion level of the cone plateau thresholds (Footnote 2). Rather, cone activity too weak to be detected alone somehow became detectable when mixed with an underlying rod signal. The marked asymmetry of the color discrimination responses suggested that the cone signal that permitted discrimination was primarily a chromatic response to the green target. Cone activity nevertheless appeared to change abruptly at the cone plateau thresholds of both targets, as evinced by the nonmonotonic brightness functions in Figure 4.

These findings can be most simply explained by the hypothesis that cone activity suppressed rod brightness signals. The only additional assumption required is that rod responses to the test targets made chromatic signals from dark-adapted cones detectable below the cone plateau threshold, but left the detectability of achromatic cone signals unchanged. Inhibition of rod-mediated brightness by achromatic cone signals then accounts for the nonadditive bright- ness matching results of Figures 2 and 3 and the brightness reversals of Figure 4, and inhibition of rod-mediated brightness by chromatic cone signals accounts for the difference between the green and white brightness matches of Figure 5a.

In summary, the present experiments show that when a homogeneous visual target stimulates both rods and cones, the cone response can reduce the rod-mediated brightness signal. This interaction can occasionally be observed directly as a reversal of the brightness vs. luminance function at the cone plateau threshold, suggesting a possible mechanism for the impairment of brightness discrimination shown by Spillmann and Breitmeyer (1977). In addition, the discovery of color discrimination well below the conventional cone plateau threshold suggests that rod responses can selectively enhance weak cone signals in chromatic neural pathways. Even these faint chromatic signals appear to measurably reduce rodmediated brightness. Possible implications of these results for other studies of low-level color discrimination, such as McCann's studies of rod contributions to color sensation (McCann, 1972; McCann \& Benton, 1969; McKee, McCann, \& Benton, 1977) remain to be investigated.

\section{REFERENCES}

Clarke, F. J. J. Extra-foveal colour metrics. Optica Acta, 1960 , 7, 355-384.

Drum, B. Forced-choice colour discrimination in the dark-adapted parafovea. In G. Verriest (Ed.), Colour vision deficiencies $V$. Bristol: Adam Hilger, 1980.

Ekman, G., \& Gustavsson, U. Threshold values and the psychophysical function in brightness vision. Vision Research, $1968,8,747-758$.

Hollins, M. Brightness contrast at low luminances. Vision Research, 1971, 11, 1459-1472.

Lowenste in, O., \& Loewenfeld, I. E. The pupil. In H. Davson (Ed.), The eye (Vol. 3): Muscular mechanisms. New York: Academic Press, 1969.

McCANn, J. J. Rod-cone interactions: Different color sensations from identical stimuli. Science, 1972, 176, 1255-1257.

MCCANN, J. J., \& Benton, J. L. Interaction of the long-wave cones and the rods to produce color sensations. Journal of the Optical Society of America, 1969, 59, 103-107.

McKee, S. P., McCAnN, J. J., \& Benton, J. L. Color vision from rod and long-wave cone interactions: Conditions in which rods contribute to multicolored images. Vision Research, 1977, 17, 175-185.

Palmer, D. A. Rod-cone mechanism underlying the Purkinje shift. Nature, 1976, 262, 601-603.

Richards, W., Luria, S. M., \& Matteson, H. H. Interacting spectral sensitivity functions obtained in a contrast situation. Vision Research, 1967, 7, 629-644.

Spillmann, L. A., \& Breitmeyer, B. G. Impairment of brightness discriminability at luminance levels near the alpha-cone break. Investigative Ophthalmology and Visual Science, 1977, 16(ARVO Supplement), 29.

Stabell, U., \& Stabell, B. Facilitation of chromatic cone activity by rod activity. II. Variation of chromatic-related cone activity. Scandinavian Journal of Psychology, 1971, 12, 168-174.

Wagner, G., \& Boynton, R. M. Comparison of four methods of heterochromatic photometry. Journal of the Optical Society of America, 1972, 62, 1508-1515. 
Walters, J. W. Metacontrast: The effects obtained with consecutively presented concentric disks and rings of different wavelengths. American Journal of Optometry and Archives of American Academy of Optometry, 1970, 47, 634-640.

WiLlmer, E. N. Low threshold rods and the perception of blue. Journal of Physiology (London), 1949, 111, 17 P.

\section{NOTES}

1. The cone response to the white test target could have selectively constricted the pupil by no more than $.2 \mathrm{~mm}$ under the conditions of the brightness matching experiment (see Lowenstein \& Loewenfeld, 1969). Assuming a beginning pupil diameter of $7 \mathrm{~mm}$, this would have been equivalent to less than a $6 \%$ reduction of pupil area. The corresponding brightness decrease would have been much less than $6 \%$, however, because such small pupil constrictions do not start until about $.5 \mathrm{sec}$ after the stimulus onset. Furthermore, even if significant brightness changes due to pupil constriction had been present, they would have had little or no effect on the brightness matches, because the test and reference targets were both viewed through the same pupil.

2. The descending limits method encouraged subjects to adopt a low threshold criterion. Comparisons with a two-alternative forced-choice method showed that the limits thresholds typically corresponded to about $40 \%$ frequency-of-seeing.

3. Even though the scotopic background was visible for "purerod" matches and invisible (though present) for "pure-cone" matches, the reference brightness appeared remarkably similar for the two conditions; after exposing only one eye to the fluorescent preadapting light, neither subject could detect a significant brightness difference when alternatively viewing the referenee with his preadapted and his nonadapted eye.

4. In two of B.D.'s sessions, the brightness matches for two reference levels straddled the cone plateau threshold; rather than explicitly presenting both levels in Figure 3, the overall average is shown. Because this procedure spuriously increased the variance of the brightness-match/rod-threshold ratios, the applicable levels within each session were averaged before computing the standard errors in Figure 3b.

5. A more extensive subsequent study (Drum, 1980) has also shown significant color discrimination below the cone plateau threshold in completely dark-adapted conditions, with a white target especially chosen to be indistinguishable to rods and cones. Also, see Stabell and Stabell (1971).

\section{APPENDIX DERIVATION OF ADDITIVITY FORMULA}

The formula for computing the additivity predictions in Figures 2 and 3 is derived as follows: Let $B_{g, R}, B_{w, C}$, and $B_{w, R}+C$ be matching brightnesses (i.e., $B_{g, R}=B_{w, C}=$ $B_{w, R}+C$ ) for rods (green target), cones (white target), and rods + cones (white target), respectively, and let $B_{g, R T}$, $B_{w, C T}$, and $B_{w, R T}$ be the corresponding threshold brightnesses. Assuming that brightness is proportional to luminance near threshold (Ekman \& Gustavsson, 1968),

$$
\begin{aligned}
B_{g, R} & =B_{g, R T}\left(L_{g, R} / L_{g, R T}\right), \\
B_{w, C} & =B_{w, C T}\left(L_{w, C} / L_{w, C T}\right),
\end{aligned}
$$

and

$$
\left(\mathrm{L}_{\mathrm{g}, \mathrm{R}} / \mathrm{L}_{\mathrm{g}, \mathrm{RT}}\right) \mathrm{B}_{\mathrm{g}, \mathrm{RT}}=\left(\mathrm{L}_{\mathrm{w}, \mathrm{C}} / \mathrm{L}_{\mathrm{w}, \mathrm{CT}}\right) \mathrm{B}_{\mathrm{w}, \mathrm{CT}},
$$

where $L_{g, R}$ is the (photopic) luminance that produces $B_{g, R}$, etc. Arbitrarily setting $B_{R T} \equiv 1$ (i.e., rod threshold is defined to have unit brightness), Equation 3 becomes

$$
B_{w, C T}=\left(L_{g, R} / L_{g, R T}\right) /\left(L_{w, C} / L_{w, C T}\right) .
$$

Considering the specific case, $B_{w} R+C$, where both rods and cones contribute to brightness, the additive prediction is

$$
\mathbf{B}_{w, R}+C=B_{w, R^{\prime}}+B_{w, C^{\prime}}
$$

where

$$
B_{w, R^{\prime}}=L_{w, R+C} / L_{w, R T}
$$

and

$$
B_{w, C^{\prime}}=B_{w, C T}\left(L_{w, R+C} / L_{w, C T}\right) .
$$

Note also that, since $B_{w, R}+C=B_{g, R}$ and $B_{g, R T}=1$, Equation 1 is equivalent to

$$
\mathbf{B}_{\mathrm{w}, \mathrm{R}+\mathrm{C}}=\mathrm{L}_{\mathrm{g}, \mathrm{R}} / \mathbf{L}_{\mathrm{g}, \mathrm{RT}} \text {. }
$$

Equations 6, 7, and 8 can now be substituted into Equation 5 , which becomes

$\mathrm{L}_{\mathrm{g}, \mathrm{R}} / \mathrm{L}_{\mathrm{g}, \mathrm{RT}}=\mathrm{L}_{\mathrm{w}, \mathrm{R}+\mathrm{C}} / \mathrm{L}_{\mathrm{w}, \mathrm{RT}}+\mathrm{B}_{\mathrm{w}, \mathrm{CT}}\left(\mathrm{L}_{\mathrm{w}, \mathrm{R}+\mathrm{C}} / \mathrm{L}_{\mathrm{w}, \mathrm{CT}}\right)$

Solving for $L_{w, R}+C / L_{w, R T}$ now gives

$$
\begin{aligned}
\mathbf{L}_{w, R}+C / L_{w, R T} \\
\quad=\left(L_{g, R} / L_{g, R T}\right) /\left[1+B_{w, C T}\left(L_{w, R T} / L_{w, C T}\right)\right],
\end{aligned}
$$

where $B_{w, C T}$ is expressed in luminance terms in Equation 4 and $L_{W, R}+C / L_{W}, R T$ is the predicted ratio of matching luminance to threshold luminance whose logarithm is plotted in the right-hand histogram of Figures $2 \mathrm{~b}$ and $\mathbf{3 b}$.

(Received for publication July 24, 1980; revision accepted January 12, 1981.) 